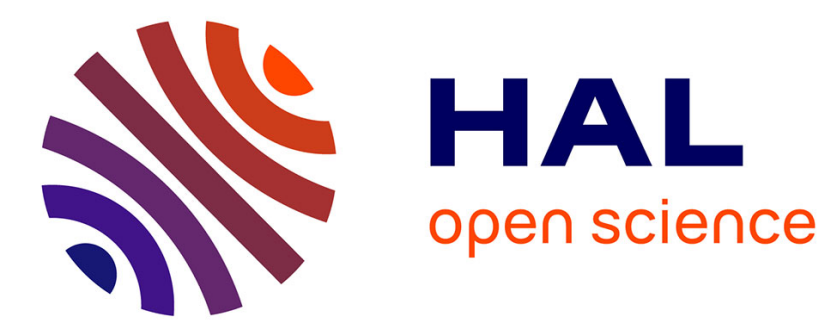

\title{
texture-based forest cover classification using random forests and ensemble margin
}

Samia Boukir, Olivier Regniers, Li Guo, Lionel Bombrun, Chrisitan Germain

\section{To cite this version:}

Samia Boukir, Olivier Regniers, Li Guo, Lionel Bombrun, Chrisitan Germain. texture-based forest cover classification using random forests and ensemble margin. IEEE International Geoscience and Remote Sensing Symposium 2015, 2015, Milan, Italy. pp.3072-3075. hal-01188174

\section{HAL Id: hal-01188174 https://hal.science/hal-01188174}

Submitted on 28 Aug 2015

HAL is a multi-disciplinary open access archive for the deposit and dissemination of scientific research documents, whether they are published or not. The documents may come from teaching and research institutions in France or abroad, or from public or private research centers.
L'archive ouverte pluridisciplinaire HAL, est destinée au dépôt et à la diffusion de documents scientifiques de niveau recherche, publiés ou non, émanant des établissements d'enseignement et de recherche français ou étrangers, des laboratoires publics ou privés. 


\title{
TEXTURE-BASED FOREST COVER CLASSIFICATION USING RANDOM FORESTS AND ENSEMBLE MARGIN
}

\author{
S. Boukir ${ }^{1}$, O. Regniers ${ }^{2}$, L. Guo ${ }^{1,2}$, L. Bombrun ${ }^{2}$, C. Germain ${ }^{2}$ \\ ${ }^{1}$ Bordeaux INP, G\&E, EA 4592, F-33600, Pessac, France \\ E-mail: name.surname@ipb.fr \\ ${ }^{2}$ CNRS - IMS Lab. (UMR 5218), University of Bordeaux, 351 Cours de la Libération, \\ 33402 Talence cedex, France \\ E-mail: name.surname@ims-bordeaux.fr
}

\begin{abstract}
This work investigates the discriminative power of wavelet decomposition based texture features in forest cover classification. Our texture features are used as inputs in a random forests classifier. The performances of this tree-based ensemble classifier are assessed by classification accuracy as well as classification confidence provided by an unsupervised version of ensemble margin. The effectiveness of the proposed texture based multiple classifier system is demonstrated in performing mapping of very high resolution forest imagery. Traditional grey level co-occurrence matrix derived texture features are also evaluated through our ensemble classification framework for comparison.
\end{abstract}

Index Terms - Texture features, wavelet decomposition, ensemble learning, ensemble margin, forest.

\section{INTRODUCTION}

Texture information is highly meaningful in land cover mapping especially in forestry [1] and its exploitation has great potential for increasing classification accuracy and confidence. Accurate classification maps are important sources of information for environmental and forest monitoring programs covering large areas. Recent studies [1, 2, 3, 4] have shown the existence of a strong relationship between forest spatial structure and texture, extracted from very high resolution images, at stand level, hence highlighting the potential of texture analysis for forest inventory applications. A forest stand would be defined by the trees and gaps that it includes. In this work, we investigate the effectiveness of wavelet decomposition based texture features for forest cover mapping. The discriminative power of these texture features is assessed in an ensemble classification framework involving Random Forests [5] and an unsupervised version of ensemble margin $[6,7]$. Random forest is one of the most powerful supervised classifiers which has been shown to be particularly successful for natural environment land cover mapping [8]. Ensemble margin provides a robust measure of classification confidence derived from the underlying voting procedure of ensemble methods [9]. We demonstrate the effectiveness of our texture based multiple classifier system in performing mapping of very high resolution forest imagery. A comparative analysis is carried out with GLCM (Grey Level Co-occurrence Matrix) derived texture features, the most commonly used for the study of forest and natural land covers [4].

\section{MATERIAL}

The Nezer forest covers about $60 \mathrm{~km}^{2}$ and is located near the Atlantic coast in South West of France within the largest European maritime pine forest. It is made up of large evenaged stands of pine-trees (4 to $50 \mathrm{ha}$ ). This study was conducted using a Quickbird image acquired simultaneously in Panchromatic (Pan, of $0.6 \mathrm{~m}$ spatial resolution) and in MultiSpectral (MS, of $2.4 \mathrm{~m}$ spatial resolution) in October 2003, covering a large forest structure diversity along the Nezer site. The multispectral image combines 4 spectral bands: Red (R), Green (G), Blue (B) and Near-Infra-Red (NIR). Two samples (of size respectively $1109 \times 1050$ pixels and $1136 \times 972$ pix$e l s$, in multispectral resolution), extracted from the Quickbird forest image (figure 1), are tested, exploiting separately MS resolution and jointly both MS and Pan resolutions. Only forest texture pixels are included in datasets, the used mask and reference stands being provided as a GIS layer. Each of the four datasets, ROI (Region Of Interest) 1 (MS and combined MS/Pan resolutions) and ROI 2 (MS and combined MS/Pan resolutions), has been divided into two equal parts, training set and test set, for supervised classification.

Five forest structure classes (figure 3(a)) have been designed to describe the forest structure diversity [4]. These different thematic classes are representative of crown size, tree density and tree spatial distribution. Tree rows are visible among the youngest stands. Irregularities in the tree spatial distribution appear in the eldest samples. The five classes have been defined by photo-interpretation. Class 1 denotes 


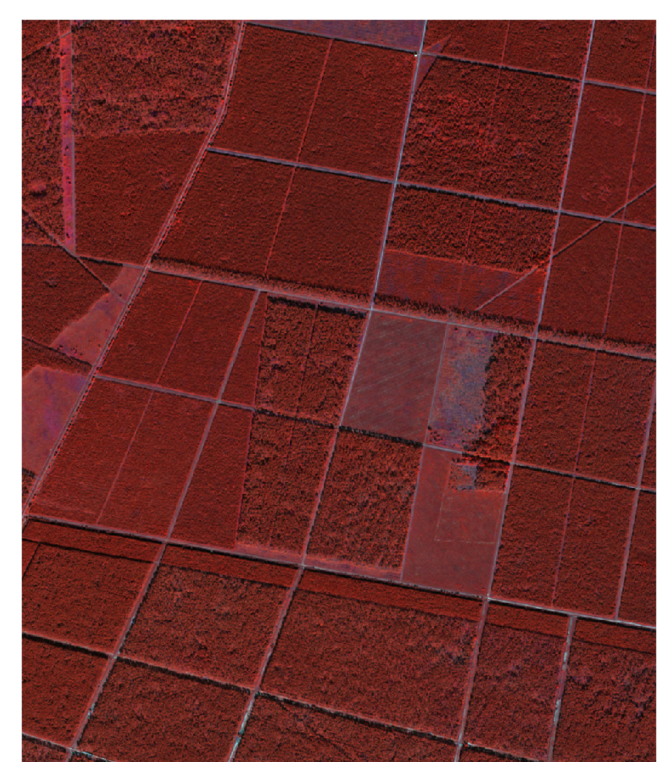

Fig. 1. Quickbird multispectral image sample (ROI2), of size $1136 \times 972$ pixels, of Nezer forest (R, G, NIR)

areas with no visible trees in the image (Cd less than $0.5 \mathrm{~m})$. This class corresponds to clear cuts or stands with very young trees (less than 3 years) where the tree height is smaller than non forest species height. Classes 2-5 correspond to different forest structure growth stages, from very regular in-line stands with high tree density and low canopies to very heterogeneous stands with low tree density and high canopies. The boundaries between all these classes are not clearly defined which makes this classification task challenging.

\section{METHODS}

In this paper, non parametric descriptors are introduced to model wavelet subbands. The resulting texture features will hence be employed in Random Forests [5], the decision tree based ensemble classifier involved in our multiple classifier framework. In literature, many authors have considered wavelet energy and wavelet entropy as texture features [10]. But none of them take into account the spatial organisation of wavelet coefficients. In a parametric context, previous works have shown that multivariate models such as Gaussian, elliptical distributions or copula based models yield a significant gain in performance compared to univariate models [1, 11, 12]. Hence, it is relevant to introduce non parametric descriptors which describe the spatial dependency of wavelet coefficients. Inspired by previous works on Mellin kind statistics for PolSAR image [13], we propose to extend this concept to derive non parametric texture features representing this spatial dependence.

Let $W_{s, o}$ be a wavelet subband at scale $s$ and orientation $o$. An array containing random vectors of spatial dependence is created by rearranging the wavelet coefficients located in a neighbourhood around the current spatial position. Covariance matrices $\Sigma_{i, j}$ are then computed by averaging those vectors in a sliding window of size $M_{x} \times M_{y}{ }^{1}$. The proposed features are obtained as the three first log-cumulants derived from the determinant of the covariance matrices located in a second sliding window of size $N_{x} \times N_{y}$ :

$$
\begin{aligned}
& \kappa_{1}=\frac{1}{N_{x} N_{y}} \sum_{i=1}^{N_{x}} \sum_{j=1}^{N_{y}} \ln \left|\Sigma_{i, j}\right| \\
& \kappa_{2}=\frac{1}{N_{x} N_{y}} \sum_{i=1}^{N_{x}} \sum_{j=1}^{N_{y}}\left(\ln \left|\Sigma_{i, j}\right|-\kappa_{1}\right)^{2} \\
& \kappa_{3}=\frac{1}{N_{x} N_{y}} \sum_{i=1}^{N_{x}} \sum_{j=1}^{N_{y}}\left(\ln \left|\Sigma_{i, j}\right|-\kappa_{1}\right)^{3}
\end{aligned}
$$

Finally, these three new features are computed for each wavelet subband resulting in a set of $3 N_{s} N_{o}$ descriptors used as input features in the random forest classifier, $N_{s}$ and $N_{o}$ being respectively the number of scales and orientations of the wavelet decomposition.

The traditional accuracy estimates are rarely accompanied by confidence limits. In our multiple classifier framework, we assess the confidence of the classification accuracy using ensemble margin [9]. Each produced classification map is associated with a margin map, introduced in previous work [14] for urban mapping using a classic ensemble margin [9] which involves the known class labels. In this work, the margin maps are produced using an unsupervised version of the classic margin we have proposed in previous work $[6,7]$. Hence, this alternative margin provides dense margin maps (confidence value for each pixel) while the classic margin leads to sparse margin maps (confidence value for each pixel with known label) [14]. The highest the margin of a sample, the more confidence in classification. Typically, the margin values would be significantly higher near class centers whereas the smaller margins would correspond mainly to class boundaries. Random Forests can produce a full classification map, in other words, a predicted class label for each instance whether it is labeled or not. Hence, the associated pair of full (dense) classification map and dense margin map can be particularly relevant in environmental and forest monitoring programs covering large areas, which require the interpretation of large volumes of remotely sensed data using typically a limited amount of reference data.

\section{RESULTS AND DISCUSSION}

A classification performance comparative analysis is conducted between the proposed wavelet based texture features and GLCM derived texture features [4]. Window size for

\footnotetext{
${ }^{1}$ This step is similar to a boxcar filter classically used in PolSAR images.
} 


\begin{tabular}{|c|c|c|c|c|}
\hline Texture & MS ROI1 & MS ROI2 & MS/Pan ROI1 & MS/Pan ROI2 \\
\hline GLCM & 77.21 & 84.08 & 89.00 & 88.97 \\
\hline Wavelets & 83.32 & 91.54 & 96.11 & 93.83 \\
\hline
\end{tabular}

Table 1. Overall classification accuracy (\%) of Random Forests using different texture analysis methods on Quickbird forest image samples (test set)

texture analysis has been set for both methods to $25 \times 25$ in MS resolution and $60 \times 60$ in Pan resolution. Both methods use a multi-scale scheme. The wavelet based texture analysis method relies on a total of 18 texture features while its GLCM counterpart involves a total of 9 texture features. Both textural and spectral (R, G, B, NIR) features are involved in the classification of two distinct samples (ROI1 and ROI2) of a Quickbird image of Nezer forest. When solely the MS resolution is considered, texture analysis is carried out on an intensity image resulting from a uniform averaging of the available multispectral bands. Random Forest decision tree ensembles were constructed with 100 trees, a typical moderate size ensemble. All the reported results are mean values of a 10-time calculation.

Table 1 shows the overall classification performance achieved by Random Forests on each of the four available datasets, ROI1 (MS and combined MS/Pan resolutions) and ROI2 (MS and combined MS/Pan resolutions), using respectively GLCM and wavelet based textural features. These results show that wavelet based features significantly outperform GLCM based features for all data sets and both MS and combined MS/Pan resolutions. The increase in classification accuracy is up to $\mathbf{7 . 5 \%}$. Unsurprisingly, the texture descriptors combining both multispectral and panchromatic resolutions are the most effective for forest texture discrimination leading to a classification accuracy of up to $96 \%$.

Figure 3 exhibits the forest cover maps provided by Random Forests using respectively GLCM derived texture features (figure 3(b)) and wavelet decomposition based texture features (figure 3(c)). These results show that our texture features outperform the traditional GLCM features in terms of mapping quality. Figure 2 shows the produced full margin map which generally exhibits a good confidence in forest texture (in-stand) classification apart from the two bottom left forest stands which belong to class 2, the smallest class (only $2.1 \%$ of training data) of this highly imbalanced data set.

\section{CONCLUSION}

We proposed a texture-based forest cover classification using random forests and ensemble margin in a very high resolution context. To this aim, non parametric features issued from Mellin kind statistics were introduced to represent the spatial dependence of wavelet coefficient. Once injected in a random forest classifier, a significant gain was obtained in comparison to classical texture features derived from GLCM.

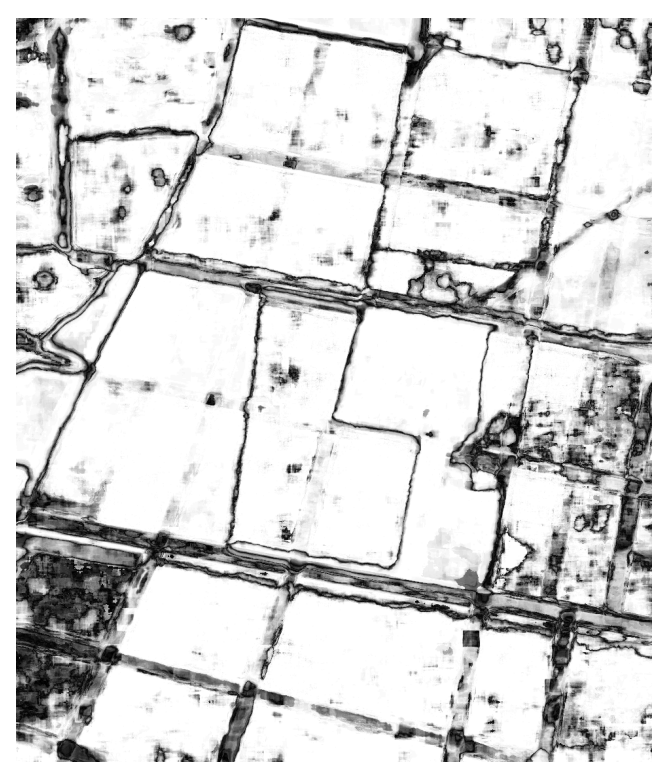

Fig. 2. Margin map of Quickbird multispectral image sample (ROI2) using texture-based random forest classification

\section{ACKNOWLEDGEMENTS}

This study was supported by $\mathrm{CNES}^{2}$ (national French space agency) and the regional council of Bordeaux (Région Aquitaine). The authors acknowledge INRA ${ }^{3}$ (French institute for agricultural research) of Bordeaux, especially Dominique Guyon and Benoit Beguet, for the VHR imagery and corresponding ground truth.

\section{REFERENCES}

[1] O. Regniers, L. Bombrun, D. Guyon, J.-C. Samalens, and C. Germain, "Wavelet-based texture features for the classification of age classes in a maritime pine forest," Geoscience and Remote Sensing Letters, IEEE, vol. 12, no. 3, pp. 621-625, March 2015.

[2] C. Song, M.B. Dickinson, L. Su, S. Zhang, and D. Yaussey, "Estimating average tree crown size using spatial information from ikonos and quickbird images: Across-sensor and across-site comparisons," Remote Sensing of Environment, vol. 114, no. 5, pp. 1099 $-1107,2010$.

[3] B. Beguet, D. Guyon, S. Boukir, and N. Chehata, "Automated retrieval of forest structure variables based on multi-scale texture analysis of vhr satellite imagery," ISPRS Journal of Photogrammetry and Remote Sensing, vol. 96, pp. 164-178, 2014.

\footnotetext{
${ }^{2}$ Centre National d'Etudes Spatiales

${ }^{3}$ Institut National de la Recherche Agronomique
} 
[4] B. Beguet, S. Boukir, D. Guyon, and N. Chehata, "Modelling-based feature selection for classification of forest structure using very high resolution multispectral imagery," in SMC'2013, Int. Conf. on Systems, Man, and Cybernetics, Manchester, UK. IEEE, 2013, pp. 4294-4299.

[5] L. Breiman, "Random forests," Machine Learning, vol. 45, no. 1, pp. 5-32, 2001.

[6] L. Guo and S. Boukir, "Margin-based ordered aggregation for ensemble pruning," Pattern Recognition Letters, vol. 34, no. 6, pp. 603-609, 2013.

[7] L. Guo, S. Boukir, and N. Chehata, "Support vectors selection for supervised learning using an ensemble approach," in ICPR'2010, 20th International Conference on Pattern Recognition. IAPR, 2010, pp. 37-40.

[8] V. F. Rodriguez-Galiano, B. Ghimire, J. Rogan, M. Chica-Olmo, and J. P. Rigol-Sanchez, "An assessment of the effectiveness of a random forest classifier for land-cover classification," ISPRS Journal of Photogrammetry and Remote Sensing, vol. 67, pp. 93-104, 2012.

[9] R.E. Schapire, Y. Freund, P. Bartlett, and W.S. Lee, "Boosting the margin: a new explanation for the effectiveness of voting methods," The Annals of Statistics, vol. 26, no. 5, pp. 1651-1686, 1998.

[10] S.E. Grigorescu, N. Petkov, and P. Kruizinga, "Comparison of texture features based on gabor filters," Image Processing, IEEE Transactions on, vol. 11, no. 10, pp. 1160-1167, Oct 2002.

[11] G. Verdoolaege and P. Scheunders, "Geodesics on the manifold of multivariate generalized Gaussian distributions with an application to multicomponent texture discrimination," International Journal of Computer Vision, vol. 95, no. 3, pp. 265-286, 2011.

[12] N.-E. Lasmar and Y. Berthoumieu, "Gaussian copula multivariate modeling for texture image retrieval using wavelet transforms," Image Processing, IEEE Transactions on, vol. 23, no. 5, pp. 2246-2261, May 2014.

[13] S. N. Anfinsen and T. Eltoft, "Application of the matrixvariate mellin transform to analysis of polarimetric radar images.," IEEE Transactions on Geoscience and Remote Sensing, vol. 49, no. 6, pp. 2281-2295, 2011.

[14] L. Guo, N. Chehata, C. Mallet, and S. Boukir, "Relevance of airborne lidar and multispectral image data for urban scene classification using random forests," ISPRS Journal of Photogrammetry and Remote Sensing, vol. 66, no. 1, pp. 56-66, 2011.

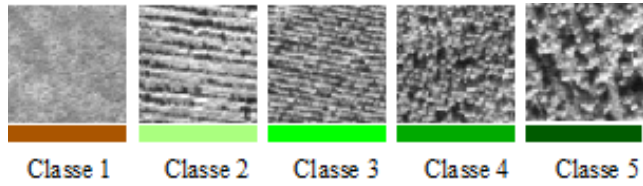

(a) Forest texture classes

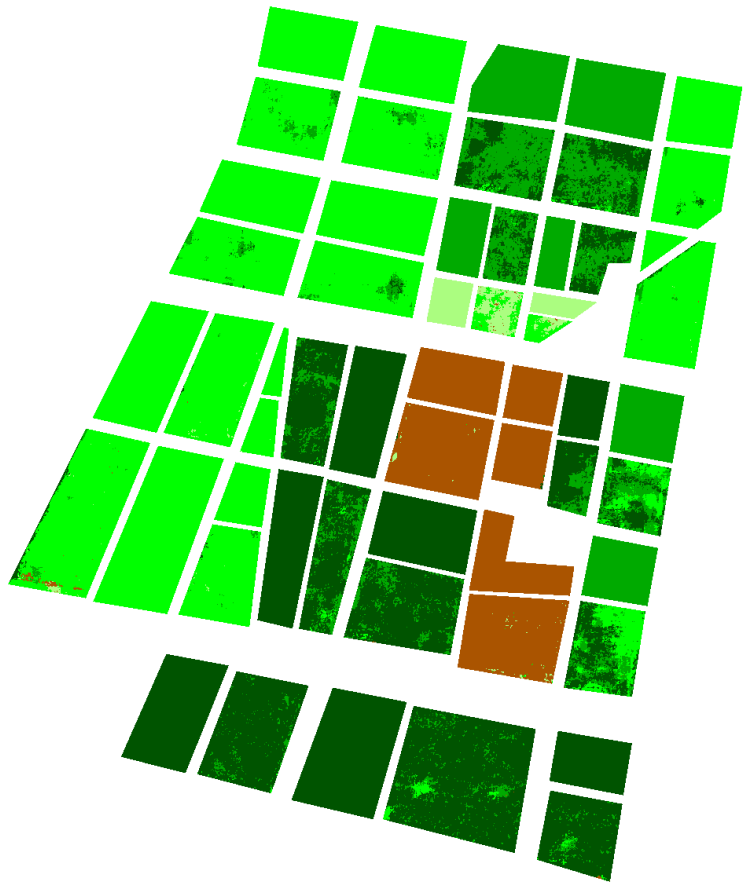

(b) Forest cover mapping with GLCM derived texture features

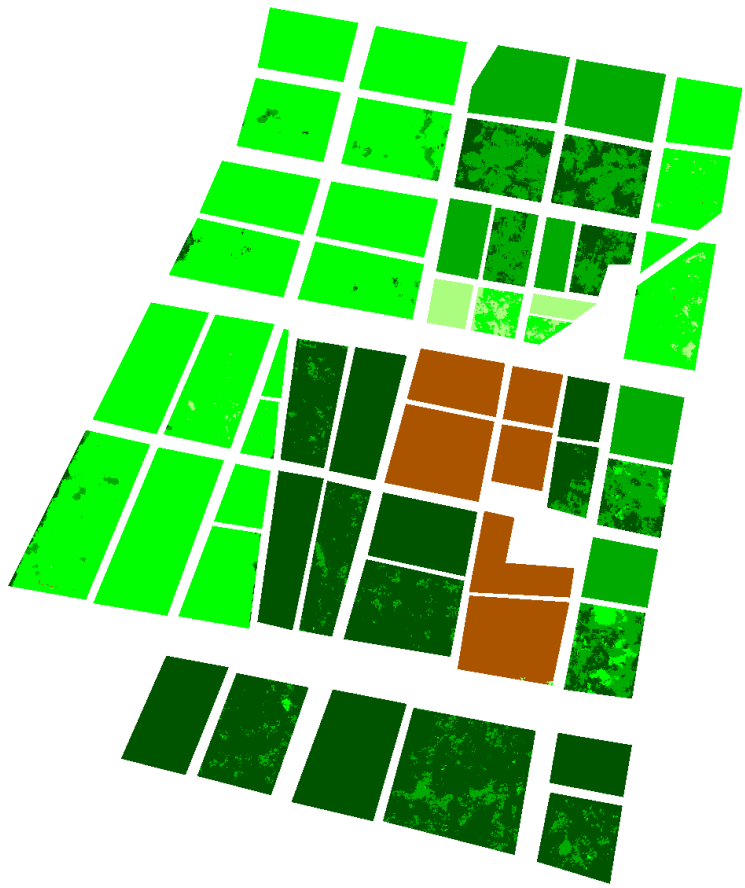

(c) Forest cover mapping with wavelet based texture features

Fig. 3. Random forest classification of Quickbird multispectral image sample (ROI2) using different texture features 